\title{
Single-cell RNA sequencing to delineate changes in tumor microenvironment induced by immunotherapy
}

\author{
*Hanne Vos ${ }^{1}$ MSc; *Junbin Qian MD, PhD², Giuseppe Floris ${ }^{3,4}$ MD, PhD; Ines Nevelsteen ${ }^{1,3} \mathrm{MD}, \mathrm{PhD}$; Kathleen Lambein ${ }^{1}$ \\ MD, PhD; Lieze Berben ${ }^{5}$ MSc; Patrick Neven ${ }^{3,6}$ MD, PhD; Hans Wildiers ${ }^{3,5}$ MD, PhD; Diether Lambrechts ${ }^{2}$ PhD, Ann \\ Smeets ${ }^{1,2} \mathrm{MD}, \mathrm{PhD}$. \\ * These authors contributed equally to this work \\ 1. Surgical Oncology, University Hospitals Leuven; Department of Oncology, KU Leuven, Leuven, Belgium \\ 2. Center for Cancer Biology, VIB, Leuven, Belgium; Laboratory for Translational Genetics, Department of \\ Oncology, KU Leuven, Leuven, Belgium \\ 3. Multidisciplinary Breast Centre, University Hospitals Leuven, Belgium \\ 4. Department of translational cell \& tissue research, University Hospitals Leuven, KULeuven; Department of \\ Oncology, KU Leuven, Leuven, Belgium \\ 5. Laboratory of Experimental Oncology, Department of General Medical Oncology, University Hospitals Leuven, \\ KU Leuven \\ 6. Department of gynaecology and obstetrics, University Hospitals Leuven, KULeuven
}

Introduction: Improved understanding of the interplay between the immune system and cancer would lead to more adequate response prediction to immune-checkpoint blockade (ICB) treatment. Furthermore, insight into immune interactions would allow identifying biomarkers for response to ICB. Single-cell RNA sequencing has emerged as a powerful technology to characterize heterogeneity in a large population of cells and opens up opportunities to predict response to therapy.

Purpose: Tracking the effect of a single dose of Pembrolizumab on the tumor microenvironment through single-cell RNA sequencing.

Methods: Single-cell RNA sequencing was performed on tumor tissue of one patient before and 10 days after a $200 \mathrm{mg}$ dose of Pembrolizumab (Keytruda ${ }^{\circledR}$ ). Changes in cell (sub)populations were analyzed. Fresh tumor material was obtained from a core needle biopsy at diagnosis and from the resection specimen. Single cell suspensions were converted to barcoded scRNA-seq libraries with the Chromium Single Cell 3' kit with 10X Genomics platform, aiming for an estimated 5,000 cells per library. The libraries were sequenced using HiSeq4000. Expression matrices were generated using CellRanger and analyzed by Seurat package. Dimensionality reduction using principle component analysis was applied to identify major cell types and their subtypes.

Results: The analyzed tumor was a grade 3 invasive ductal adenocarcinoma, hormone receptor negative and HER2+ positive, pT2NOM0. Tumor infiltrating lymphocyte (TIL) count was $30 \%$ on core biopsy and $50 \%$ on resection specimen. We sequenced a total of 9867 transcriptomes at single cell resolution before and after Pembrolizumab treatment, consisting of 5808 and 4049 cells, respectively. Major cell types of the tumor microenvironment were identified by leveraging singlecell transcriptomics analysis. We observed a sharp decrease in cancer cells after treatment $(74 \%$ versus $25 \%$ ), which was accompanied by an increase of tumor infiltrating T cells ( $18 \%$ versus $50 \%$ ). The residual cancer cells after immunotherapy showed a higher expression of the major histocompatibility complex (MHC), MHC-II in particular. In addition, we found enrichment of B-cells and endothelial cells and a downregulation of fibroblasts and myeloid cells. Furthermore, we analyzed subtypes of each cell type. We found a marked increase in cytotoxic CD4 (6\% versus 18\%) and cytotoxic CD8 cells ( $4 \%$ versus $12 \%$ ) in the T cell population. These cytotoxic CD8 cells clearly expressed higher PD-1 after immunotherapy. A decrease in CD4 Tregs, naïve CD4 and intermediate CD8 cells was observed and the $B$ cell enrichment after treatment was mainly driven by the increase of follicular B-cells. The increase of endothelial cells was driven by capillary tumor endothelial but not tip cell population, suggesting vessel normalization rather than neo-vascularization. 
Conclusion: Single-cell RNA sequencing provides a powerful tool in detecting changes in the tumor microenvironment induced by immunotherapy, and thus offers new opportunities to predict response to immunotherapy. Thirty-three additional patient samples will be analyzed in the near future, with a special focus on T- and B- cell receptor repertoire. 